\title{
Versorgungsforschung: Erfahrungen bei der Planung und Umsetzung einer Studie mit nichtakademischen, ambulanten Studienzentren
}

\section{Healthcare Research: Experience in the Planning and Implementation of a Study with Non-Academic, Outpatient Study Centres}

Autoren

Norman Best ${ }^{1,2}$, Dana Loudovici-Krug1

Institute

1 Institut für Physiotherapie, Universitätsklinikum Jena, Jena

2 Posture and Motion Group, Universitätsklinikum Jena, Jena

\section{Schlüsselwörter}

Gesundheitsforschung, Ambulante klinische Studie, Versorgungsrealität, Studienplanung, nicht-universitäre Forschung

Key words

health research, outpatient clinical study, supply reality, study planning, non-university research

online publiziert $\quad 16.06 .2020$

Bibliografie

Akt Rheumatol 2021; 46: 474-478

DOI 10.1055/a-1158-8586

ISSN 0341-051X

C 2020. Thieme. All rights reserved.

Georg Thieme Verlag KG, Rüdigerstraße 14,

70469 Stuttgart, Germany

Korrespondenzadresse

Dr. Norman Best

Institut für Physiotherapie

Universitätsklinikum Jena

Erlanger Allee 101

07740 Jena

Tel.: 036419325226 , Fax: 036419325202

Norman.Best@med.uni-jena.de

\section{ZUSAMMENFASSUNG}

Hintergrund Realistische Zahlen zur Versorgungsrealität in Deutschland sind wichtig, wenn es um Verteilung von Ressourcen im Gesundheitssystem geht. Studien, die realistische Kennziffern zu bestimmten Fragestellungen und Diagnosen liefern, müssen gefördert werden.
Ziel der Arbeit Es sollte mittels geeignetem Studiendesign über eine breite Masse an teilnehmenden Arzt- und Physiotherapiepraxen ( $n=96)$ ein hoher Stichprobenumfang $(n=1000)$ zur Korrelation von Funktionsparametern des Bewegungssystems generiert werden.

Material und Methoden Klassische Befunde sollten mittels Fragebogen zur Studienzentrale gelangen. Dies war sowohl analog als auch digital möglich. Der Zeitaufwand lag für die prognostizierte Anzahl von 10 Datenblättern pro Studienzentrum bei ca. 60 min. insgesamt.

Ergebnisse Ernüchternd war die initiale Teilnehmerzahl von 6 Praxen. Nur nach erneuter Erinnerung mittels Motivationsschreiben konnte die Teilnehmerzahl auf 19 gesteigert werden. Es wurden 423 Rückläufer registriert. Trotz lediglich eines einfachen Einschlusskriteriums, wurden 92 Bögen zwar vollständig, aber dennoch fehlerhaft zugesandt.

Schlussfolgerung Um die Teilnahmebereitschaft zu erhöhen reicht die angenommene intrinsische Motivation nicht aus. Wichtiger erscheint es, konkret auf einzelne, interessierte Teilnehmer zuzugehen und sie von der Bedeutung der Studie zu überzeugen. Klarheit in Struktur und Ablauf sind entscheidend, da Versorgungsforschung für die direkten Akteure zusätzlich zum ärztlichen und therapeutischen Tagesgeschäft und zumeist unentgeltlich erbracht wird.

\section{ABSTRACT}

Background Realistic statistics on the reality of healthcare in Germany are important when it comes to the distribution of resources in the healthcare system. Studies that provide reliable indicators for specific questions and diagnoses must be promoted.

Objective A large sample size $(n=1000)$ for the correlation of functional parameters of the musculoskeletal system was to be generated by means of a suitable study design over a broad mass of physicians' and physiotherapists' practices $(n=96)$.

Material and methods Characteristic findings were to be sent to the study centre by questionnaire. This was possible both analogously and digitally. The overall time required for the predicted number of 10 data sheets per study centre was approximately 60 minutes. 
Results The initial number of only 6 participating practices was disillusioning. Only after a reminder was sent with a motivational letter could the number of participants be increased to 19. There were 423 registered returns. Although there was only one simple inclusion criterion, 92 returned sheets were complete, but incorrect.
Conclusion The assumed intrinsic motivation is not sufficient to increase the willingness to participate. It seems to be more important to approach individual interested participants and convince them of the importance of the study. Clarity in structure and procedure is crucial since healthcare research for the direct players is provided in addition to the daily medical and therapeutic business and mostly free of charge.

\section{Hintergrund}

Wissenschaftliches Arbeiten umfasst ein großes Spektrum an Möglichkeiten. Viele Kolleginnen und Kollegen in der Medizin sind initial davon abgeschreckt, dass ein scheinbar hohes zusätzliches Arbeitspensum und eine kaum zu durchschauende Statistik auf sie warten. Häufig werden multizentrische, epidemiologische Studien mit erheblichem finanziellem und organisatorischem Aufwand als Goldstandard der Wissenschaft angesehen. Auch im Bereich der Physikalischen Medizin und Rehabilitation (PRM) sind solche Studien häufig anzutreffen $[2,4,7]$. Dabei ist es jedoch so, dass v. a. in kleinen Fachgebieten bislang viele Themen wenig bis gar nicht bearbeitet wurden und hier wissenschaftlich mit einfachen Fragestellungen begonnen werden kann. So ist eine Befragung hausärztlich tätiger Kollegen zur Versorgungsrealität nicht zwingend uninteressanter, als große epidemiologische Studien. Aus Sicht der Autoren gibt es im Bereich der PRM viele unbeantwortete Fragen. Beispielsweise ist unklar, wie häufig Patienten mit speziellen Erkrankungen in den Sprechstunden der PRM-Fachärzte vorstellig werden, welche Zeitressourcen zur Behandlung tatsächlich genutzt werden müssen und Ähnliches.

Hier soll nicht der Fragebogen, dessen Anwendung und Ergebnis vorgestellt und diskutiert werden. Vielmehr werden Schwierigkeiten bei der Planung einer wissenschaftlichen Untersuchung in der Versorgungsforschung benannt. Das Ziel der Arbeit ist es somit, Hinweise für die Durchführung zukünftiger Forschungsarbeit zu eröffnen.

\section{Material und Methoden}

\section{Studiendesign und Untersuchungsmethoden - Design der initial geplanten Studie}

Durch die initial geplante Studie sollte die Frage beantwortet werden, ob und wenn ja welche Zusammenhänge beim Auftreten eines sogenannten Vorlaufs eines Sakroiliakalgelenks (SIG) bei Untersuchung im Stehen nachweisbar sind. Die Prüfung auf das Vorliegen eines SIG-Vorlaufs ist ein Standardtest, welcher in der manualmedizinischen Untersuchung Erfahrenen keine Schwierigkeiten bereitet. Um diese Frage fachlich korrekt zu beantworten, entschlossen sich die Studienautoren, ausschließlich Lehrende sowie Leitende eines der Ausbildungsseminare der Deutschen Gesellschaft für Manuelle Medizin (DGMM) als Untersuchungsdurchführende auszuwählen. Es wurde angenommen, dass die intrinsische Motivation zur Studienteilnahme der manualmedizinischen/-therapeutischen Lehrenden hoch sei. Es wird von Physiotherapeutlnnen und ÄrztInnen, die in der Lehre der manuellen Therapie/Medizin stark einge- bunden sind, häufig moniert, dass valide Daten zum Auftreten verschiedener Symptome, so auch dem SIG-Vorlauf, fehlen würden. Durch die Studienautoren wurde ein Fragebogen entwickelt, der im Vorfeld mit einigen zufällig ausgewählten manualmedizinischen Lehrenden besprochen und auf (Praxis-)Tauglichkeit geprüft wurde. Der zeitliche Aufwand zum Ausfüllen des Bogens belief sich auf ca. eine Minute. Die aufzunehmenden Daten sind Routinedaten im Rahmen der manualmedizinischen Untersuchung. Ein positives Votum der zuständigen Ethikkommission der Friedrich-Schiller-Universität Jena liegt vor.

Bei ca. 100 Lehrenden und einer geplanten Rücklaufquote von ca. 10 Bögen pro Lehrenden, wurde mit einer auszuwertenden Stichprobe von ca. 1000 Datenblättern gerechnet. Es wurden möglichst viele Wege zur Datenübertragung offeriert, um die Ausbilder für eine Teilnahme zu gewinnen. Dabei waren vorgesehen: Zusendung der Bögen über Fax oder E-Mail, direktes Ausfüllen einer aktiven PDF-Vorlage, Einwählen über einen QR-Code in einen eigens eingerichteten Cloud-Ordner für direktes Onlineausfüllen und natürlich das Zusenden per Post.

Im Rahmen einer Lehrertagung, bei dem verpflichtend alle Lehrenden dieses Ausbildungsseminars anwesend sein mussten, wurden Briefe verteilt, in denen zum Thema hingeführt sowie Hintergrund und Sinn der Studie dargelegt wurden. Weiterhin konnten Zeitaufwand, Rücksendungsumfang und mögliche Wege zur Rücksendung dem Schreiben entnommen werden.

Einziges Einschlusskriterium war ein positiver Vorlauf des SIG auf einer Seite im Stehen. Trat dieses Symptom auf, wurden die Patienten in die Studie eingeschlossen und weitere übliche manualmedizinische Untersuchungen erfolgten.

In einer Studienzentrale sollte die Auswertung mit gängigen statistischen Methoden durchgeführt werden, auf welche an dieser Stelle nicht näher eingegangen wird, da das Ziel dieser Übersicht nicht die eigentliche Studie zum SIG-Vorlaufphänomen ist. Die Datenaufnahme wurde über einen Zeitraum von ca. 5 Monaten geplant.

\section{Tatsächlicher Studienablauf}

Wie oben geschildert, wurden die Lehrenden zum Zeitpunkt einer Lehrertagung zur Studienteilnahme eingeladen. Unmittelbar nach Beginn der Datenaufnahme erreichten die ersten Auswertungsbögen die Studienzentrale. Es fiel auf, dass trotz des formulierten Einschlusskriteriums, eine relativ große Anzahl von Bögen eintraf, die dieses nicht beachteten. Gegen Ende der initial geplanten Datenaufnahme konnten lediglich von 6 manualmedizinisch Lehrenden die Zusendungen registriert werden. Positiv anzumerken ist, dass von einigen, der beteiligten Studienpraxen, mehr Bögen als gefor- 
dert zugesandt wurden. Aufgrund der deutlich reduzierten Teilnehmerzahl erfolgte eine Erinnerung an Durchführung und Wichtigkeit der Studie über ein erneutes Anschreiben ca. 2 Monate nach Studienstart. Dadurch ließen sich weitere KollegInnen zur Teilnahme motivieren. Dennoch zeigte sich eine erhebliche Diskrepanz zwischen erwarteter und tatsächlicher Teilnehmer- bzw. Rücklaufquote.

- Abb. 1. zeigt den zeitlichen Studienablauf. In > Abb. 2 und $\mathbf{3}$ sind die geplanten vs. tatsächlichen Kennzahlen der Studie darge-

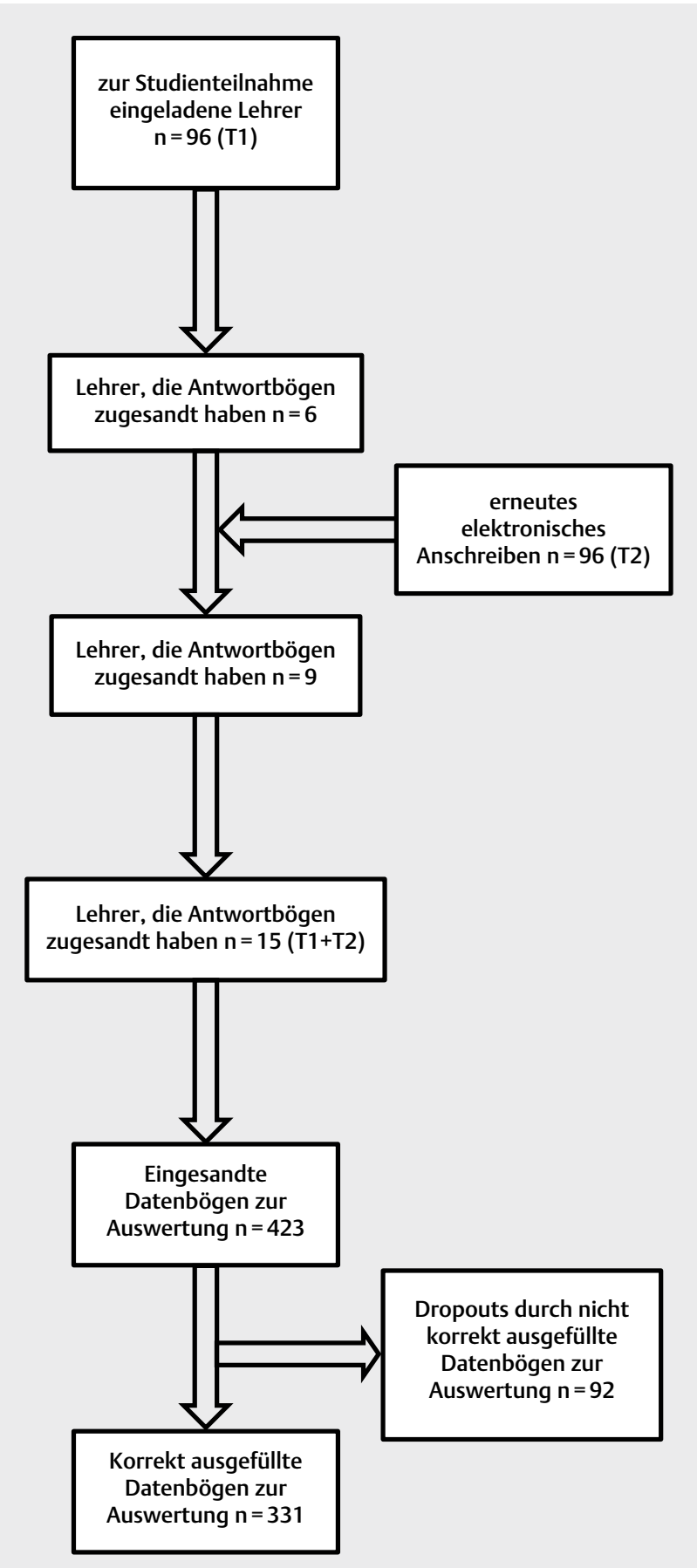

- Abb.1 Zeitlicher Ablauf der Teilnehmerakquise.

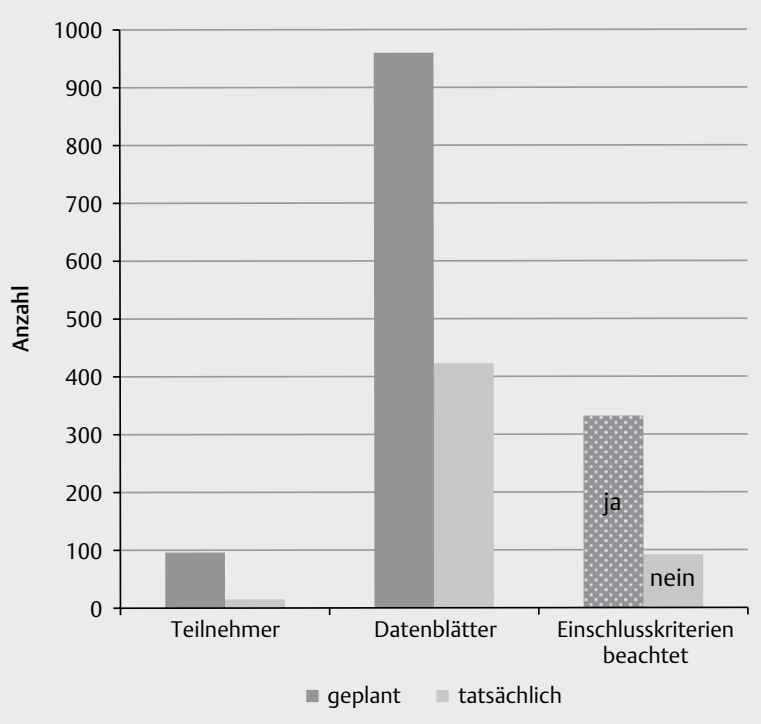

- Abb.2 Gegenüberstellung geplanter zu tatsächlicher Studienteilnahme.

stellt bzw. die zugesandten Datensätze der einzelnen Studienzentren aufgelistet.

Letztlich wurden 2 Wege zur Übermittlung der Datensätze an die Studienzentrale genutzt. Die meisten Kollegen übersandten die Bögen in Papierform. Einige wenige Teilnehmer, die, die über ein Sekretariat verfügten, schickten Scans der zuvor analog erfassten Untersuchungsbögen via E-Mail. Kein einziger Eingang konnte via Cloud, Onlineausfüllung oder über andere digitale Kanäle registriert werden.

Überraschenderweise wurden die Datensätze direkt nach Studienbeginn bis auf eine Ausnahme ausschließlich von ärztlichen Kollegen zugesandt. Im Gegensatz dazu wurde zuvor angenommen, dass primär Datenbögen aus den physiotherapeutischen Praxen das Studienzentrum zur statistischen Auswertung erreichen würden. Grundannahme war, dass durch den strukturierten Behandlungskontakt zum Patienten, die Akquise von Daten einfacher sei, als in einer ärztlichen Sprechstunde. Jedoch konnte nach erneuter Aufforderung die Zahl der teilnehmenden Physiotherapeuten auf 7 gesteigert werden. Damit entfallen knapp 2 Drittel der Teilnehmer auf ÄrztInnen. Eine noch größere Diskrepanz ist zu erkennen, wenn man die Gesamtanzahl der zugesandten Datensätze nach beiden Berufsgruppen unterteilt. Lediglich ca. 15\% der eingereichten Bögen stammen von Physiotherapeutlnnen.

\section{Diskussion}

Es ist bekannt, dass die Datenakquise mittels Fragebogen mit Dropouts behaftet ist. Hippler beschreibt bereits 1988 Rücksenderaten bei postalischen Befragungen zwischen 10 und $90 \%$ [3]. Porst bestätigt dies in seiner Veröffentlichung und rät deswegen zu prägnanten Fragestellungen. Diese könnten die Rückläuferquote deutlich erhöhen [5]. Es ist ebenfalls unbestritten, dass im Rahmen von Anfragen per E-Mail die Rücklaufquote davon abhängt, welche Größe die Befragung misst und ob der Absender weiblich oder 


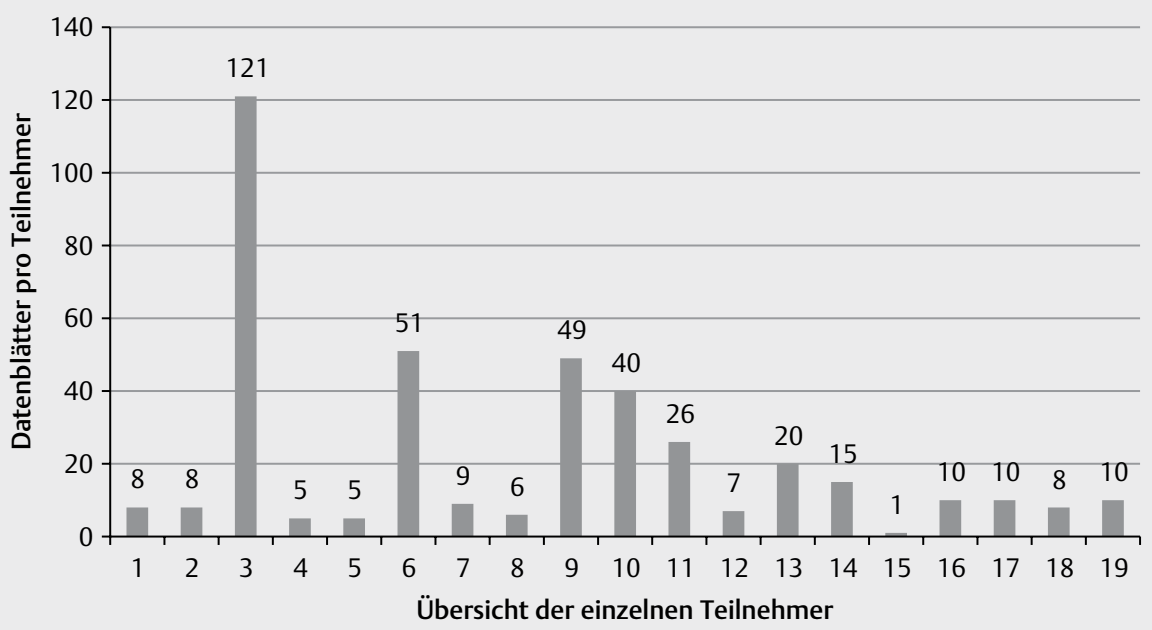

- Abb. 3 Übersicht zur Datenübermittlung einzelner Teilnehmer.

männlich ist [1]. Das bedeutet also, dass die Motivation der potenziellen Antwortgeber extrem wichtig für die erfolgreiche Durchführung einer Fragebogenumfrage ist. Dies heißt, übertragen auf das initial geplante Studiendesign, dass nicht ausschließlich Fragen beantwortet werden sollen, sondern der potenzielle Teilnehmer zusätzlich aktiv werden muss (bzgl. Datenübermittlung etc.) und somit die notwendige intrinsische Motivation deutlich höher anzusetzen ist, als in einer reinen Befragung.

Zurbuchen et al. befragten chirurgische, nicht-universitäre Einrichtungen, nach deren Interesse zur Teilnahme an multizentrischer Forschung, Studienerfahrung sowie strukturellen Gegebenheiten und konnten eine Rücklaufquote von $54 \%$ erreichen. Aus Sicht der Autoren ist dies ein eher geringer Wert, da gerade im chirurgischen Fachbereich über die Administration der Kliniken, auch ein monetäres Interesse besteht, evidenzbasierte Methodik gegenüber Kostenträgern geltend zu machen [8]. Im vorliegenden Fall kann ein monetärer Hintergrund als Motivation ausgeschlossen werden. Hier besteht primär das Interesse am Nachweis für die Sinnhaftigkeit des eigenen professionellen Handelns. Die Studienautoren vertraten vor Durchführung der Studie die Ansicht, dass es jedem manualmedizinisch tätigen Lehrer wichtig sein müsse, zur Evidenzbildung funktioneller Zusammenhänge beizutragen. Diese Annahme kann hier als nicht zutreffend deklariert werden. Letztlich ließen sich nur einige wenige Kollegen zur Teilnahme bewegen, obwohl die zur Teilnahme angefragten manuellen Ausbilder den Studienautoren persönlich bekannt waren. Die in universitären Einrichtungen tätigen ÄrztInnen waren führend bei der Datenakquise. 83 Datensätze wurden allein von einem Kollegen aus dem universitären Umfeld erbracht. Dabei waren Einrichtungen, die einen alleinigen Versorgungsauftrag haben, gar nicht oder nur sehr spärlich vertreten. Bei der Planung von Versorgungstudien sollte die positive Wirkung der intrinsischen Motivation zu einer Studienteilnahme nicht überschätzt werden.

Einen nicht geringen technischen Aufwand stellte die Bereitstellung digitaler und analoger Zugangswege zum Studienzentrum dar. Leider blieben die modernen digitalen Verfahren ungenutzt.
Im Rahmen einer studentischen Prüfungsvorbereitung zeigten sich andere Werte. Dabei nutzten mehr als 3 Viertel der befragten Studierenden ausschließlich digitale Zugänge. Niemand bereitete sich ausschließlich klassisch bzw. analog vor [6]. Nun könnte man den Studierenden zugutehalten, dass diese jünger und damit technisch versierter als die Teilnehmer der hier vorgestellten Studie seien. Aus Sicht der Autoren kann ein Alters- oder Wissensnachteil weitestgehend verneint werden. Es scheint jedoch so zu sein, dass die papierhafte Dokumentation schneller und unkomplizierter umzusetzen ist. Das Versenden per Post war für die Teilnehmenden finanziell dabei irrelevant.

\section{Schlussfolgerung}

Es empfiehlt sich die Reduktion der Anzahl von beteiligten Studienteilnehmern auf wenige. Zu Beginn der Erarbeitung des Studiendesigns sollte ein Studientreffen anberaumt werden, bei dem alle potenziellen Studienpraxen über die Wichtigkeit und den Ablauf aufgeklärt werden. Hierbei sollte ganz klar aufgeführt werden, welchen zeitlichen Rahmen die Datenakquise tatsächlich einnimmt und welchen konkreten Nutzen der Teilnehmer davon hat. Möglicherweise ist es sinnvoll, Mentoren zu benennen, die eine kleine Gruppe von Studienpraxen betreuen und ständigen Kontakt mit diesen halten. Aus Sicht der Autoren besteht so die Möglichkeit, die Rücksendebereitschaft bzw. Teilnahme zu erhöhen. Es kann nicht ausschließlich aus berufspolitischer Sicht auf die intrinsische Motivation gesetzt werden. Versorgungsforschung, auch wenn diese von extern geplant ist, wird von den direkten Akteuren zusätzlich zum ärztlichen und therapeutischen Tagesgeschäft und des Weiteren zumeist unentgeltlich erbracht. Daher ist die Überzeugungsarbeit vor Beginn der Datenaufnahme entscheidend.

\section{Interessenkonflikt}

Die Autorinnen/Autoren geben an, dass kein Interessenkonflikt besteht. 


\section{Literatur}

[1] Greif $\mathrm{V}$, Batinic B. Die Bedeutung des Einladungsschreibens für die Rücklaufquote in Online-Befragungen. In: Jahrbuch der Absatz- und Verbrauchsforschung. GfK Nürnberg: Gesellschaft für Konsum-, Markt- und Absatzforschung e.V; 2007: 162-177

[2] Hass HG, Rockstroh J, Pech I et al. Medizinisch-beruflich orientierte Rehabilitation (MBOR) in der Onkologie - 2-Jahres-Erfahrungen mit einem standardisierten, multimodalen Therapiekonzept. Phys Med Rehab Kuror 2018; 28: 341-346

[3] Hippler HJ. Methodische Aspekte schriftlicher Befragungen: Probleme und Forschungsperspektiven. planung und analyse 1988; 6: 244-248

[4] Kessemeier FM, Stöckler C, Petermann F et al. Psychometrische Eigenschaften der Readiness for Return to Work Scale für Patienten in der psychosomatischen Rehabilitation. Phys Med Rehab Kuror 2017; 27: $335-347$
[5] Porst R. Thematik oder Incentives? Zur Erhöhung der Rücklaufquoten bei postalischen Befragungen. ZUMA Nachrichten 1999; 23: 72-87

[6] Romeike B, Petersen I. Stellenwert der digitalen Mikroskopie im Rahmen der Vorbereitung und Durchführung der Prüfung allgemeine Pathologie. In: Gemeinsame Jahrestagung der Gesellschaft für Medizinische Ausbildung (GMA) und des Arbeitskreises zur Weiterentwicklung der Lehre in der Zahnmedizin (AKWLZ). Leipzig: German Medical Science GMS; 2015: 53-54

[7] Zein K, Saal S. Ambulante Nachsorge von chronischen Schlaganfallpatienten mit Physiotherapie, Ergotherapie und Logopädie. Phys Med Rehab Kuror 2017; 27: 275-284

[8] Zurbuchen U, Schwenk W, Bussar-Maatz R et al. Klinische Studien außerhalb von Universitätskliniken. Der Chirurg 2010; 81: 160-166 